\title{
Frecuencias de grupos sanguíneos e incompatibilidades $A B O$ y RhD, en La Paz, Baja California Sur, México
}

Lorenzo del Peón-Hidalgo, Biól, ${ }^{(1)}$ Ma Guadalupe Pacheco-C ano, Q F, ${ }^{(2)}$ Mirna Zavala-Ruiz, MP, ${ }^{(2)}$ Alejandro Madueño-López,T éc Lab, ${ }^{(2)}$ A dolfo García-González, Dr en C.

Del Peón-Hidalgo L, Pacheco-Cano MG, Zavala-Ruiz M, Madueño-López A, García-González A. Frecuencias de grupos sanguíneos e incompatibilidades $A B O$ y RhD,

en La Paz, Baja California Sur, México.

Salud Publica Mex 2002;44:406-412.

El texto completo en inglés de este artículo está disponible en: http://www.insp.mx/salud/index.html

\section{Resumen}

Objetivo. D eterminar las frecuencias génicas y de fenotipo, y predecir el riesgo de incompatibilidad y aloinmunización materna en la población de La Paz, Baja California Sur, México. Material y métodos. Estudio descriptivo en el que se evaluaron 1809 disponentes sanguíneos altruistas que acudieron en 1998 al Hospital General de Zona, del Instituto Mexicano del Seguro Social en La Paz, Baja California Sur, México, tipificados por aglutinación en tubo. Las frecuencias génicas fueron estimadas asumiendo condiciones de equilibrio, y las incompatibilidades y riesgo de aloinmunización de acuerdo con la literatura. Los datos fueron evaluados estadísticamente mediante $\chi^{2}$. Resultados. La población reveló las siguientes frecuencias en porcentaje: $0,58.49 ; A, 31.40 ; B, 8.40 ; A B, 1.71 ; R h D, 95.36 ; y$ RhD negativo, 4.64 y las frecuencias génicas fueron $i, 0.7648 ; \mathrm{A}, 0.1821$; $I^{B}, 0.0519 ; D, 0.7845 ; d, 0.2155$, respectivamente. Las incompatibilidades en parejas y maternofetal fueron 0.3023 y 0.1685 para $A B O, 0.0442$ y 0.0364 para RhD y 0.0134 y 0.0061 para incompatibilidad doble, respectivamente. La probabilidad de aloinmunización materna fue estimada en 0.0309 . Conclusiones. En La Paz, los grupos 0 y RhD son los más abundantes, aunque las frecuencias están entre las más bajas en México, contrario a lo ocurrido paraA y RhD negativo. La probabilidad de aloinmunización materna y las incompatibilidades son también elevadas. Los grupos ancestrales blanco, negro e indio interactuaron en la zona no-
Del Peón-Hidalgo L, Pacheco-Cano MG, Zavala-Ruiz M, Madueño-López A, García-González A. Blood group $A B O$ and $R h D$ frequencies and incompatibilities

in La Paz, Baja California Sur, Mexico.

Salud Publica Mex 2002;44:406-412.

The English version of this paper

is available at: http://www.insp. $\mathrm{mx} / \mathrm{salud} / \mathrm{index} . \mathrm{html}$

\begin{abstract}
A bstract
Objective.To determine genic and phenotypic frequencies and predict the risk of incompatibility and maternal alloimmunization in the population of La Paz. Material and Methods. This descriptive study evaluated 1809 voluntary blood donors attending in 1998 the Hospital G eneral de Zona of Instituto Mexicano del Seguro Social (Zone General Hospital of the Mexican Institute of Social Security) in La Paz, Baja California Sur, Mexico. Blood donors were typified by tube agglutination. The gene frequencies were estimated assuming equilibrium conditions, and incompatibilities and allo immunization were statistically assessed with the $\chi^{2}$ test. Results. Percent frequencies were as follows: blood group $0,58.49 \% ; A, 31.40 \% ; B, 8.40 \% ; A B, 1.71 \% ; R h D, 95.36 \%$; and RhD negative, $4.64 \%$. Genic frequencies were: $i, 0.7648 ; I^{A}$, $0.1821 ; I^{B}, 0.0519 ; D, 0.7845 ;$ and $d, 0.2155$, respectively. Incompatibilities between couples and mother-child were 0.3023 and 0.1685 for $A B O, 0.0442$ and 0.0364 for RhD, and 0.0134 and 0.0061 for double incompatibility, respectively. The probability of maternal alloimmunization was estimated at 0.0309 . Conclusions The 0 and RhD groups were the most common in La Paz, although frequencies were among the lowest in Mexico, contrary to the case of $A$ and RhD negative groups. The probabilities of maternal alloimmunization and of incompatibilities were also high. Ancestral white, black, and indigenous groups admixed in the northwestern part of Mexico; after migrating to Baja Cali-
\end{abstract}

(1) Coordinación D elegacional. Instituto Mexicano del Seguro Social (IMSS). Baja California Sur, México.

(2) Hospital General de Zona N 0. 1. IMSS. Baja California Sur, México.

Fecha de recibido: 5 de marzo de 2002 - Fecha de aprobado: 25 de mayo de 2002

Solicitud de sobretiros: Lorenzo del Peón-Hidalgo. Coordinación de Investigación Médica. Jefatura de Prestaciones Médicas. Instituto Mexicano del Seguro Social. Calle Ignacio Allende N 0.1115, Colonia Centro. 23000 La Paz, BCS, México.

Correo electrónico:Iph5502@ prodigy.net.mx 
roeste del país, determinando al migrar a Baja California Sur un mestizaje probablemente similar al resto de la zona noroeste. El texto completo en inglés de este artículo está disponible en: http://www.insp.mx/salud/index.html

Palabras clave: grupos sanguíneos; incompatibilidad de grupos sanguíneos; México fornia Sur the admixture of the population probably became similar to that of the remainder of the northwestern area.The English version of this paper is available at: http:// ww w.insp. $\mathrm{mx} / \mathrm{salud} /$ index.html

Key words: blood group; blood group incompatibility; Mexico
E 1 conocimiento de los grupos sanguíneos ha con tribuido al entendimiento de algunos de los mecanismos básicos de la herencia, y a un siglo de que Landsteiner ${ }^{1}$ los descubriera siguen siendo de gran interés práctico y conceptual. Las frecuencias de los grupos sanguíneos de los sistemas $\mathrm{ABO}$ y $\mathrm{RhD}$ han sido estudiadas a escala mundial. En nuestro país, los estudios de Lisker y colaboradores ${ }^{2-5}$ aportan información sobre las frecuencias $\mathrm{ABO}$ y $\mathrm{RhD}$ de las zonas centro y sur -y los de Garza-Chapa y colaboradores ${ }^{6,7}$ para las zonas norte y nordeste.

La posibilidad de relacionar la presencia de determinado grupo sanguíneo con alguna patología ha sido siempre tentadora. En estudios de hace más de una década, ${ }^{8,9}$ algunos de ellos controversiales, se pretendió relacionar problemas de esterilidad en la pareja matrimonial con incompatibilidad ABO. Otros estudios ${ }^{10-12}$ relacionaron incompatibilidad $\mathrm{ABO}$ entre parejas con la presencia de abortos repetitivos.

Los grupos sanguíneos ABO y RhD juegan un importante papel en obstetricia. Las madres RhD negativas, al ser sensibilizadas por antígenos eritrocitarios de un producto $\mathrm{RhD}$, producirán anticuerpos Anti-D que al cruzar la barrera placentaria pueden hemolizar los eritrocitos fetales causando la anemia hemolítica del recién nacido. ${ }^{13}$ Los casos de incompatibilidad fetomaterna en nuestro país ${ }^{14,15}$ constituyen un importante factor de riesgo perinatal que, de acuerdo con lo observado en poblaciones mestizas, disminuye conforme aumenta la contribución génica indígena. ${ }^{16}$

El grupo sanguíneo $\mathrm{ABO}$ puede influir también sobre la aloinmunización Rh. Se ha demostrado que existe un mayor número de casos de sensibilización a $\mathrm{Rh}$ cuando se presentaban hemorragias transplacentarias durante el embarazo o cuando los cónyuges pertenecen a grupos sanguíneos $\mathrm{ABO}$ compatibles. ${ }^{17,18}$ Los mecanismos que explican el fenómeno fueron propuestos desde los años sesenta. ${ }^{19-21}$ Finn $^{22}$ sugiere por primera vez la posibilidad de impedir la inmunización materna destruyendo con anticuerpos los eritrocitos fetales presentes en la circulación de la madre, apli- cándole $\gamma$-globulina anti-D al momento del parto, lo cual fue demostrado posteriormente por varios grupos. ${ }^{23-26} \mathrm{~A}$ finales de la década quedó claramente establecida la utilidad del tratamiento. ${ }^{27}$

En nuestro país la prevención de la inmunización materna es insuficiente según lo demostraron Zavala y Salamanca ${ }^{28}$ basados en la comparación entre el número estimado de madres en riesgo, dentro del Instituto Mexicano del Seguro Social (IMSS), con las dosis de $\gamma$-globulina anti-D adquiridas por la misma institución.

La población indígena en la península de Baja California, al establecerse las misiones hacia el año de $1697,29-31$ estaba integrada por los grupos Cochimí, Guaycura y Pericú, y contaba con 40000 o 50000 individuos. El régimen misional, la escasez de alimentos, la guerra y las enfermedades introducidas diezmaron la población en menos de un siglo, estimándose que en 1762 vivían en las misiones 2059 indígenas ${ }^{32}$ y sólo 700 u 800 blancos.$^{31,32}$ La población indígena disminuyó hasta desvanecerse, en la primera mitad del siglo XIX, ${ }^{33}$ entre los nuevos inmigrantes que a partir de entonces repoblarían la península.

Durante el México independiente se inicia el reparto de tierra en la península con fines ganaderos y posteriormente agrícolas, todo favorecido por el establecimiento de rutas comerciales con el resto del país; esto tuvo como consecuencia la migración de nuevos grupos de población, fundamentalmente de la misma zona noroeste. ${ }^{34}$ La población actual de La Paz presenta algunas características importantes: $33.77 \%$ tuvo su lugar de residencia anterior fuera del estado, ${ }^{34}$ de ellos, 35\% provino de la misma región noroeste: Baja California (14\%), Sinaloa (13.86\%), Sonora $(7.49 \%)$ y, en menor proporción, de otros estados como Jalisco $(7.49 \%)$ y Durango (5.2\%).

Los objetivos de nuestro estudio en la población de La Paz incluyen la determinación de las frecuencias fenotípica y génica de los sistemas sanguíneos $\mathrm{ABO}$ y RhD, la estimación de la frecuencia de las incompatibilidades $\mathrm{ABO}, \mathrm{RhD}$ y dobles en las parejas 
matrimonial y feto-materna, así como calcular el riesgo y número de mujeres que pueden sufrir aloinmunización.

\section{Material y métodos}

A partir de las libretas de registro del Banco de Sangre del Hospital General de Zona No. 1 del IMSS, en La Paz Baja California Sur, se obtuvieron los datos referentes a fecha, nombre, grupo $\mathrm{ABO}$ y $\mathrm{Rh}$, sexo, y lugar de residencia de las personas que durante 1998 acudieron a donar sangre. Las pruebas de tipificación sanguínea de los disponentes fueron realizadas por el personal del Banco de Sangre.

El grupo sanguíneo ABO fue determinado por aglutinación en tubo, mediante las pruebas directa e inversa. En la prueba directa, los antígenos A y B de los eritrocitos se identificaron, mediante aglutinación en tubo, con reactivos hemoclasificadores comerciales de tipo monoclonal (Anti-A, anti-B y anti-AB; Gamma Biological Inc. Houston TX US). En la prueba inversa, la presencia de anticuerpos anti-A y anti-B en el suero del disponente fue revelada por la aglutinación en tubo de los eritrocitos de grupos sanguíneos conocidos, suministrados rutinariamente por el Banco Central de Sangre del Centro Médico Nacional Siglo XXI del IMSS, en México D. F. El antígeno D de los eritrocitos del disponente fue identificado, por aglutinación en tubo, mediante la prueba directa, con reactivo hemoclasificador monoclonal (Anti-D, Gamma Biological Inc. Houston TX US). La negatividad del RhD se confirmó, por medio de la prueba de antiglobulina humana, empleando un reactivo monoclonal comercial (anti-IgG-C3d; Gamma Biological Inc. Houston TX US), descartando así la posibilidad de que se tratara de células $\mathrm{D}^{\mathrm{U}}$.

El grupo sanguíneo, sexo o estrato social del disponente no constituyeron criterios de selección para el banco de sangre. Fueron aceptados todos los disponentes, la mayoría altruistas, derechohabientes o no del IMSS que cumplieron los requisitos establecidos por la Norma Oficial para tales casos. ${ }^{35}$ Después de seleccionar a las personas radicadas en la ciudad de La Paz, y de eliminar los registros duplicados, se incluyeron en el estudio los datos de 1809 donadores, 1622 hombres $(89.66 \%)$ y 187 mujeres $(10.34 \%)$. Las frecuencias fenotípicas entre ambos grupos no mostraron diferencias significativas, por lo que se consideraron un solo grupo.

La frecuencia de cada uno de los fenotipos se determinó expresándola en porcentaje con respecto al total de individuos estudiados. El cálculo de las frecuencias génicas se realizó asumiendo que la población se encuentra en condiciones de equilibrio, según lo establecido por Hardy ${ }^{36}$ y Weinberg. ${ }^{37}$ La frecuencia del gen $\mathrm{O}(\mathrm{i}=\sqrt{(0)})$ se calculó como la raíz cuadrada de la frecuencia de individuos de la población pertenecientes al grupo sanguíneo $\mathrm{O}$; la frecuencia del gen $\mathrm{A}\left(\mathrm{I}^{\mathrm{A}}=1-\sqrt{B+0}\right)$, restando a la unidad la raíz cuadrada de la suma de las frecuencias de individuos con fenotipos $\mathrm{B}$ y $\mathrm{O}$; y la frecuencia del gen $\mathrm{B}\left(\mathrm{I}^{\mathrm{B}}=1-\sqrt{A+0}\right)$, restando de la unidad la raíz cuadrada de la suma de las frecuencias de individuos con fenotipos A y O. Por su parte, la frecuencia génica $d$ se calculó al obtener la raíz cuadrada de la frecuencia de individuos con fenotipo $\mathrm{RhD}$ negativo $(\mathrm{d}=\sqrt{\mathrm{R} h \mathrm{Dneg}})$; y la frecuencia génica de $D(D=1-d)$ se obtuvo restando de la unidad la frecuencia génica $\mathrm{d}^{6}{ }^{6}$

Las frecuencias de las incompatibilidades fetomaterna y matrimonial en $\mathrm{ABO}, \mathrm{RhD}$ o a ambos son estimaciones de tipo teórico, basadas en las frecuencias de los grupos sanguíneos de una población y fueron calculadas de acuerdo con Garza-Chapa y colaboradores. ${ }^{6} \mathrm{La}$ incompatibilidad $\mathrm{ABO}$ entre matrimonios (IM) se obtuvo como $\mathrm{IM}=[\mathrm{O}(\mathrm{A}+\mathrm{B}+\mathrm{AB})+\mathrm{A}(\mathrm{B}+\mathrm{AB})+$ $\mathrm{B}(\mathrm{A}+\mathrm{AB})]$, donde $\mathrm{O}, \mathrm{A}$ y $\mathrm{B}$ representan las frecuencias fenotípicas de estos grupos. La incompatibilidad $\mathrm{ABO}$ feto-materna (IFM) se obtuvo como IFM= $\left[\mathrm{I}^{\mathrm{A}}(\mathrm{B}+\mathrm{O})+\right.$ $\left.\mathrm{I}^{\mathrm{B}}(\mathrm{A}+\mathrm{O})\right]$, donde $\mathrm{I}^{\mathrm{A}} \mathrm{e}^{\mathrm{B}}$ representan las frecuencias de los genes A y B, y A, B y O, las frecuencias fenotípicas de estos grupos. La incompatibilidad del RhD en matrimonios se obtuvo multiplicando la frecuencia fenotípica de individuos $\mathrm{RhD}$ por la frecuencia de los $\mathrm{RhD}$ negativo, en tanto que la incompatibilidad fetomaterna del $\mathrm{RhD}$ se calculó como ( $=\mathrm{D}(\mathrm{RhD}$ negativo), donde $\mathrm{D}$ representa la frecuencia del gen $\mathrm{D}, \mathrm{y} \mathrm{RhD}$ negativo, la frecuencia de individuos con ese fenotipo. La incompatibilidad doble en matrimonios se obtuvo al multiplicar las incompatibilidades $\mathrm{ABO}$ y $\mathrm{Rh}$ en las parejas; de igual forma, la doble incompatibilidad feto-materna fue calculada al multiplicar la incompatibilidad feto-materna $\mathrm{ABO}$ por la incompatibilidad feto-materna $\mathrm{RhD}$. El cálculo de las incompatibilidades para otras ciudades del país, se estimó a partir de datos reportados referentes a la distribución de los tipos sanguíneos $\mathrm{ABO}$ y $\mathrm{Rh}$ en esas poblaciones.

El número de mujeres en riesgo de sensibilización en la población de La Paz se obtuvo de la suma de partos y abortos anuales multiplicados por la probabilidad de estar en riesgo, restándole el porcentaje de las que ya estuvieran inmunizadas (3.2\% para población del IMSS),${ }^{14}$ de acuerdo con Zavala y Salamanca. ${ }^{28}$

El riesgo de sufrir aloinmunización que tiene una madre perteneciente a la población de La Paz se obtuvo multiplicando la probabilidad de ser $\mathrm{RhD}$ negativo por la probabilidad de tener un producto RhD y por la 
probabilidad de que madre e hijo sean compatibles $\mathrm{ABO}{ }^{28}$

Por otro lado, se estimó el riesgo que tienen las madres Rh negativas de sufrir aloinmunización, clasificándolo como riesgo alto, cuando los tipos sanguíneos de madre y producto son $\mathrm{ABO}$ compatibles $\mathrm{y}$, como riesgo bajo, cuando no lo son. En ambos casos, el cálculo se efectuó multiplicando la frecuencia del grupo sanguíneo de la madre por la frecuencia del grupo sanguíneo del producto. ${ }^{15}$

Para determinar el equilibrio de Hardy-Weinberg en la población estudiada, y para estimar la similitud de las frecuencias ABO de la ciudad de La Paz respec- to a otras ciudades del país, se empleó la prueba estadística de $\chi^{2}$.

\section{Resultados}

Los valores de las frecuencias observadas en la población de La Paz, en cuanto al sistema $\mathrm{ABO}$ se refiere, no difieren significativamente $\left(\chi^{2}=0.3695, p>0.05\right)$ de aquellos esperados bajo las condiciones de equilibrio propuestas por Hardy y Weinberg.

El cuadro I muestra la distribución de fenotipos y frecuencias génicas de los sistemas sanguíneos $\mathrm{ABO}$ y Rh en la población de La Paz. El grupo O fue el más

\section{Cuadro I}

Frecuencias fenotípicas y génicas de Los sistemas sanguíneos ABO y RhD en La Paz, Baja California Sur, México, 1998

\begin{tabular}{|c|c|c|c|c|c|c|c|c|c|c|}
\hline \multicolumn{7}{|c|}{$\mathrm{ABO}$} & \multicolumn{4}{|c|}{$\operatorname{Rh}(D)$} \\
\hline \multicolumn{4}{|c|}{ Frecuencia fenotípica (\%) } & \multicolumn{3}{|c|}{ Frecuencia génica } & \multicolumn{2}{|c|}{ Frecuencia fenotípica (\%) } & \multicolumn{2}{|c|}{ Frecuencia génica } \\
\hline A & B & $A B$ & 0 & $I^{A}$ & $\mathrm{I}^{\mathrm{B}}$ & $\mathrm{i}$ & Positivo & N egativo & D & $d$ \\
\hline 31.0 & 8.40 & 1.71 & 58.49 & 0.1821 & 0.0519 & 0.7648 & 95.36 & 4.64 & 0.7845 & \\
\hline
\end{tabular}

Valores obtenidos de los donadores ( $N=1809$ ) del banco de sangre durante 1998 en el Hospital General de Zona N 0. 1 de La Paz, Baja California Sur

\section{Cuadro II \\ EstimACIÓN DE LAS FRECUENCIAS (\%) DE INCOMPATIBILIDAD FETO-MATERNA, MATRIMONIAL Y DOBLE PARA LOS GRUPOS sanguíneos ABO y Rh en La Paz, Baja California Sur y otras ciudades, México, 1998}

\begin{tabular}{|c|c|c|c|c|c|c|c|}
\hline \multirow[b]{2}{*}{ Ciudad* } & \multirow[b]{2}{*}{ Estado } & \multicolumn{2}{|c|}{ Incompatibilidad ABO } & \multicolumn{2}{|c|}{ Incompatibilidad Rh } & \multicolumn{2}{|c|}{ Incompatibilidad doble } \\
\hline & & Matrimonios & Feto-maternà & Matrimonios & Feto-maternā & Matrimonios & Feto-materna \\
\hline Monterrey & Nuevo León & 32.09 & 18.27 & 7.11 & 5.56 & 2.28 & 1.02 \\
\hline Zacatecas & Zacatecas & 30.43 & 16.90 & 4.66 & 3.82 & 1.42 & 0.64 \\
\hline Veracruz & Veracruz & 27.82 & 15.48 & 4.57 & 3.75 & 1.27 & 0.58 \\
\hline La Paz & Baja California Sur & 30.23 & 16.85 & 4.42 & 3.64 & 1.34 & 0.61 \\
\hline Guadalajara & Jalisco & 31.31 & 17.47 & 4.12 & 3.41 & 1.29 & 0.60 \\
\hline México & Distrito Federal & 25.76 & 14.23 & 3.93 & 3.27 & 1.01 & 0.47 \\
\hline Paraíso & Tabasco & 21.12 & 10.92 & 3.84 & 3.20 & 0.81 & 0.35 \\
\hline Mérida & Yucatán & 26.65 & 14.32 & 3.75 & 3.13 & 1.00 & 0.45 \\
\hline Durango & Durango & 29.60 & 16.29 & 3.28 & 2.77 & 0.97 & 0.45 \\
\hline Saltillo & Coahuila & 28.23 & 15.37 & 3.00 & 2.55 & 0.85 & 0.39 \\
\hline Ciudad Victoria & Tamaulipas & 27.78 & 15.41 & 2.82 & 2.41 & 0.78 & 0.37 \\
\hline El Carmen & Campeche & 24.38 & 13.60 & 2.72 & 2.33 & 0.66 & 0.32 \\
\hline 0 axaca & 0 axaca & 23.40 & 12.24 & 2.63 & 2.26 & 0.61 & 0.28 \\
\hline Saladero & Veracruz & 30.13 & 16.06 & 2.44 & 2.10 & 0.73 & 0.34 \\
\hline Gómez Palacio & Durango & 31.57 & 17.51 & 2.06 & 1.80 & 0.65 & 0.31 \\
\hline León & Guanajuato & 26.85 & 15.71 & 1.86 & 1.64 & 0.50 & 0.26 \\
\hline Torreón & Coahuila & 26.74 & 14.37 & 1.77 & 1.56 & 0.47 & 0.22 \\
\hline Puebla & Puebla & 23.13 & 12.39 & 1.77 & 1.56 & 0.41 & 0.19 \\
\hline Tlaxcala & Tlaxcala & 23.41 & 13.11 & 0.70 & 0.64 & 0.16 & 0.08 \\
\hline
\end{tabular}

* La estimación de las incompatibilidades se calculó basándose en las frecuencias poblacionales ABO y RhD reportadas en el cuadro III 
abundante $(58.49 \%)$ en las personas estudiadas. La frecuencia del grupo A fue también notable (31.40\%); por el contrario, los grupos B $(8.40 \%)$ y AB $(1.71 \%)$ estuvieron menos representados. Las frecuencias génicas respectivas fueron 0.7648 para $\mathrm{O}$, para $\mathrm{A}, 0.1821$ y para $\mathrm{B}$, 0.0519. En cuanto al sistema $\mathrm{Rh}, 95.36 \%$ de las personas tipificadas fueron $\mathrm{RhD}$ y $4.64 \% \mathrm{RhD}$ negativas. Las frecuencias génicas $\mathrm{D}$ y d fueron estimadas en 0.7845 y 0.2155 , respectivamente.

Las incompatibilidades feto-materna y la matrimonial debidas a $\mathrm{ABO}$ revelaron una frecuencia de 16.85 y $30.23 \%$, respectivamente las debidas a $\mathrm{RhD} 3.64$ y $4.42 \%$, y la doble incompatibilidad ABO-RhD $0.61 \mathrm{y}$ $1.34 \%$, respectivamente (cuadro II).

La distribución de frecuencias $\mathrm{ABO}$ en diversas ciudades de la República muestra al grupo O como el más frecuente. La frecuencia del grupo $\mathrm{O}$, si bien es la mayor en La Paz (58.49\%), se encuentra entre las más bajas en México. Por el contrario, la frecuencia del grupo A, menos abundante que el $\mathrm{O}$ en $\mathrm{La} \mathrm{Paz}(31.4 \%)$, es una de las más altas reportadas en el ámbito nacional. De manera similar, el grupo $\mathrm{RhD}$ negativo $(4.6 \%)$ con menor frecuencia en La Paz se ubica entre los más altos en el país (cuadro III).

Para la ciudad de La Paz, el riesgo que tiene una madre de sufrir aloinmunización fue de 0.0309 y se obtuvo de multiplicar la probabilidad de ser RhD negativo (0.0464) por la de tener un producto $\mathrm{RhD}$ (0.9536) (cuadro I) y por la probabilidad de que madre y producto sean un par compatible en ABO (0.6977) (cuadro IV).

El $69.77 \%$ de las mujeres $\mathrm{RhD}$ negativo con productos RhD (cuadro IV) se estima puedan tener productos compatibles en $\mathrm{ABO}$, por tanto, mayor riesgo de aloinmunización. El 30.23\% restante corresponde a mujeres con productos $\mathrm{RhD}$ de grupo sanguíneo $\mathrm{ABO}$ no compatible y con menor riesgo de sufrir aloinmunización.

El número de madres en riesgo de sufrir aloinmunización, durante 1998, en la ciudad de La Paz fue de 140 (cuadro V).

\section{Discusión}

Al comparar las frecuencias de grupos sanguíneos $\mathrm{ABO}$ y $\mathrm{RhD}$ en la población de La Paz con el promedio estimado a partir de reportes de otros autores en el ámbito nacional, se observa que el grupo $\mathrm{O}$, presente en $58.49 \%$ de las personas, tiene una distribución considerablemente menor al $65 \%$ estimado como promedio $(p<0.05)$; por el contrario, el grupo A, presente en $31.4 \%$ de las personas, exhibió una frecuencia mayor

Cuadro III

Frecuencias fenotípicas (\%) ABO y RHD REPORTADAS PARA DIVERSAS CIUDADES deL PAís

\begin{tabular}{|c|c|c|c|c|c|c|c|c|}
\hline Ciudad & Estado & 0 & A & B & $A B$ & $\chi^{2 *}$ & Rhd & Referencia \\
\hline La Paz & Baja California Sur & 58.49 & 31.4 & 8.40 & 1.71 & 0 & 4.64 & - \\
\hline Guadalajara & Jalisco & 57.2 & 31.2 & 9.7 & 1.9 & 4.6 & 4.3 & 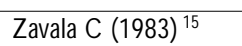 \\
\hline Gómez Palacio & Durango & 57.99 & 29.17 & 10.76 & 2.08 & 17 & 2.08 & Garza R (1984) ${ }^{6}$ \\
\hline Ciudad Victoria & Tamaulipas & 63.6 & 27.3 & 7.4 & 1.7 & 19.9 & 2.9 & Zavala C (1996) ${ }^{28}$ \\
\hline Monterrey & N uevo León & 63.1 & 26.5 & 9.0 & 1.4 & 22.1 & 7.7 & Garza R (1978) ${ }^{7}$ \\
\hline Veracruz & Veracruz & 64.2 & 25.7 & 8.1 & 2.0 & 29.9 & 4.8 & Lisker R (1986) ${ }^{3}$ \\
\hline Saltillo & Coahuila & 64.2 & 24.9 & 9.7 & 1.2 & 40.7 & 3.1 & Lisker R $(1990)^{5}$ \\
\hline Saladero & Veracruz & 60.5 & 28.6 & 10.9 & 0.0 & 50 & 2.5 & Lisker R $(1986)^{3}$ \\
\hline Torreón & Coahuila & 66.35 & 24.47 & 8.3 & 0.88 & 53.6 & 1.76 & Garza R $(1984)^{6}$ \\
\hline México & Distrito Federal & 67.7 & 23.4 & 7.2 & 1.7 & 66.15 & 4.1 & Zavala C (1983) ${ }^{15}$ \\
\hline Durango & Durango & 55.1 & 38.6 & 6.3 & 0.0 & 73.7 & 3.4 & Zavala C (1996) ${ }^{28}$ \\
\hline El Carmen & Campeche & 69.7 & 22.0 & 6.4 & 1.8 & 98.4 & 2.8 & Lisker R $(1986)^{3}$ \\
\hline Mérida & Yucatán & 67.5 & 21.1 & 10.5 & 0.9 & 102.5 & 3.9 & Lisker R $(1990)^{5}$ \\
\hline León & Guanajuato & 65.3 & 24.7 & 6.0 & 4.0 & 108.9 & 1.9 & Lisker R $(1990)^{5}$ \\
\hline Zacatecas & Zacatecas & 61.9 & 22.2 & 13.5 & 2.4 & 113.6 & 4.9 & Reveles F(1987) ${ }^{39}$ \\
\hline Tlaxcala & Tlaxcala & 71.7 & 19.6 & 6.5 & 2.2 & 144.5 & 0.7 & Crawford M (1974) ${ }^{40}$ \\
\hline Puebla & Puebla & 72.3 & 19.5 & 7.4 & 0.8 & 151.2 & 1.8 & Lisker R $(1988)^{4}$ \\
\hline 0 axaca & 0 axaca & 71.8 & 20.5 & 7.7 & 0.0 & 155 & 2.7 & Lisker R $(1990)^{5}$ \\
\hline Paraíso & Tabasco & 75.8 & 14.9 & 9.3 & 0.0 & 281.9 & 4.0 & Lisker R $(1986)^{3}$ \\
\hline Promedio & & 65.0 & 25.0 & 8.6 & 1.4 & & 3.7 & \\
\hline
\end{tabular}




\section{Cuadro IV}

\section{EsTIMACIÓN DEL RIESGO DE INMUNIZACIÓN MATERNA en ReLACión con el sistema ABO. La Paz, Baja California Sur, México, 1998}

\begin{tabular}{|c|c|c|c|}
\hline $\begin{array}{c}\text { Madre } \\
\text { (RhD negativo) } \\
\text { Grupo sanguíneo }\end{array}$ & $\begin{array}{c}\text { Hijo } \\
\text { (RhD) } \\
\text { Grupo sanguíneo }\end{array}$ & $\begin{array}{c}\text { Riesgo alto } \\
\text { (ABO compatibles) } \\
(\%)\end{array}$ & $\begin{array}{c}\text { Riesgo bajo* } \\
\text { (ABO no compatibles) } \\
(\%)\end{array}$ \\
\hline 0 & 0 & 34.21 & \\
\hline 0 & A & & 18.37 \\
\hline 0 & $B$ & & 4.91 \\
\hline 0 & $A B$ & & 1.00 \\
\hline A & 0 & 18.37 & \\
\hline A & A & 9.86 & \\
\hline A & B & & 2.64 \\
\hline A & $A B$ & & 0.54 \\
\hline$B$ & 0 & 4.91 & \\
\hline$B$ & A & & 2.64 \\
\hline$B$ & $B$ & 0.71 & \\
\hline$B$ & $A B$ & & 0.14 \\
\hline$A B$ & 0 & 1.00 & \\
\hline$A B$ & A & 0.54 & \\
\hline$A B$ & $B$ & 0.14 & \\
\hline$A B$ & $A B$ & 0.03 & \\
\hline Suma & & 69.77 & 30.23 \\
\hline
\end{tabular}

\section{Cuadro V}

Número de MADRES EN RIESGO DE SUFRIR aloinmunización en La Paz, Baja California Sur, MéxıICO, 1998

\begin{tabular}{lrrrc} 
Hospital & Partos & Abortos & Partostabortos & En riesgo \\
IMSS* & 1756 & 193 & 1949 & 58 \\
\hline SSA $^{\ddagger}$ & 2083 & 185 & 2268 & 68 \\
\hline ISSSTE $^{\S}$ & 418 & 60 & 478 & 14 \\
Total & 4257 & 438 & 4695 & 140
\end{tabular}

* Coordinación Delegacional de Informática Médica.

₹ Sistema de Información en Salud para Población A bierta.

$\S$ Sistema de Ingresos y Egresos Hospitalarios.

IMSS: Instituto Mexicano del Seguro Social SSA: Secretaría de Salud

ISSSTE: Instituto de Seguridad y Servicios Sociales de losTrabajadores del Estado al promedio de $25.0 \%(p<0.05)$. La negatividad del RhD para La Paz (4.6\%) también fue mayor que 3.1\% promedio para el país $(p<0.05)$. De igual forma, la probabilidad de aloinmunización de 0.0309 en La Paz es mayor que la de 0.0247 obtenida por Zavala y colaboradore ${ }^{28}$ para las madres mexicanas, sin embargo, esta diferencia no es significativa.

Sin ser regla, los grupos sanguíneos A y RhD negativo se encuentran más ampliamente distribuidos en poblaciones de las zonas norte y noroeste del país (Durango, La Paz, Guadalajara, Monterrey), mientras que en poblaciones del sur y sureste, (Paraíso, Tlaxcala, Puebla y Oaxaca) presentan frecuencias considerablemente menores (cuadro III).

Lisker señala al grupo indígena como el principal contribuyente en el modelo trihíbrido (indio, blanco y negro) en la mayoría de las comunidades estudiadas, y correlaciona distintos resultados con eventos sociales, históricos y geográficos que los explican. Lo anterior da idea de lo complejo y variado que puede llegar a ser el proceso de mestizaje.

La población de la zona noroeste no es la excepción y, aunque históricamente estuvieron presentes las tres principales etnias ancestrales, la proporción con la que cada uno de los grupos contribuyó al mestizaje en esta población aún no ha sido determinada; sin embargo, no parece demasiado arriesgado pensar que el grupo indígena, por ser el más abundante en la zona (a pesar de haberse extinguido en la península), haya influido más que el grupo blanco, y éste que el grupo negro ${ }^{38}$ que ocupaba los últimos estratos sociales.

La población autóctona de La Paz no tuvo muchas oportunidades de contribuir al mestizaje actual; sin embargo, existe similitud (cuadro III) entre las frecuencias sanguíneas observadas y las reportadas para otras ciudades del país donde aún existen grupos indígenas. Esto puede explicarse si los inmigrantes que repoblaron la península durante el siglo XX, y se asentaron en La Paz por ser este el principal puerto de arribo, tuvieran ya frecuencias sanguíneas similares a las actuales.

\section{Agradecimientos}

A los doctores Jorge Arellano-Blanco y Eugenio Aguilar-Parada por sus comentarios e inapreciables consejos. Al personal del Hospital General de Zona No. 1 del Instituto Mexicano del Seguro Social por todas las facilidades prestadas. 


\section{Referencias}

1. Landsteiner $\mathrm{K}$. U eber agglutinationserscheinurgen normalen menschilchen blutes. W ien Klin W schr 1901;14:1132-1134.

2. Lisker R. Estructura genética de la población mexicana. México, D.F.: Salvat Mexicana de Ediciones, 1981.

3. Lisker R, BabinskyV.Admixture estimates in nine Mexican Indian groups and five east coast localities. Rev Invest Clin 1986;38:145-149.

4. Lisker R, Pérez-Briceño R, Granados J, BabinskyV. Gene frequencies and admixture estimates in the State of Puebla, Mexico.Am J Phys Anthropol 1988;76:331-335

5. Lisker R, Ramírez E, Pérez-Briceño R, G ranados J, Babinsky V. Gene frequencies and admixture estimates in four Mexican Urban Centers. Hum Biol 1990;62:791-801.

6. Garza-Chapa R, Tobías-Chávez R, Cerda-Flores R, Leal-Garza C. Los grupos sanguíneos $A B O$ y $R h(D)$ en poblaciones de la Región Lagunera México (Cálculo de la frecuencia de incompatibilidad simple y doble en matrimonios y maternofetal). Salud Publica Mex 1984;26:130-137.

7. G arza-C hapa R, G onzález-Rendón M, Joffre G. G rupos sanguíneos A BO y $\mathrm{Rh}^{0}$ (D) en poblaciones del IMSS en el estado de Nuevo León. Arch Invest Med (Mex) 1978;9:541-558.

8. Solish G, Gershowitz H. Distribution of the ABO blood types among fertile and infertile women. Am J Hum G enet 1969;21:23-35.

9. Kucher AN , Dubvova IE, Kuvatoba O L, Altukhov IP. Population genetic study of differential fertility in man.IV. Distribution of blood groups and frecuency of incompatible marriages. Genetika 1987;23:1671-1683.

10. Peritz E.A statistical study of the intrauterine selection factors related to $A B O$ systems. II.The analysis of foetal mortality data. Ann Hum Genet 1971;34:389-394.

11. Carapella-de Luca E, Purpura M, Coghi Y, N icotra M, Bottini E. Blood groups and histocompatibility antigens in habitual abortion. Hematologia 1980;13:105-111

12. Bottini E, Bonci E, Gloria-Bottini F, N icotra M, Carapella E, Coghi Y et al. MN Ss genetic polymorphism and intrauterine selection through habitual abortion. Hum Biol 1987:59:103-120.

13. Levine P, Katzin E, Burnha M. Isoimmunization pregnancy: Its posible bearing on erythroblastosis fetalis. J Am Med Assoc 1941:116:825-827.

14. Instituto Mexicano del Seguro Social. Investigación clínica y epidemiológica, IMSS. Los factores de riesgo perinatal en la población adscrita al Instituto Mexicano del Seguro Social. México, D.F.: IMSS, 1977.

15.Zavala C, Velázquez-Ferrari MA, N avarrete $C$, Rosales-C orona J, Lisker R. Estimation of the number of females at risk of isoinmmunization to the $\mathrm{Rh}(\mathrm{D})$ antigen in a sample of the population attended at the Instituto Mexicano del Seguro Social.Arch Invest Med 1983;14:199-206.

16. Garza-C hapa R, Rojas-Alvarado MA. Risk estimation of $A B O$ and $R h$ (D) incompatibility in persons with mono and polyphyletic surnames in Monterrey, Mexico. Comparison with other Mexican populations. Arch Med Res 1996;27:243-253.

17. Levine P.The influence of the ABO system on Rh hemolytic disease. Hum Biol 1958:30:14-27.

18. N evalinna HR, Vainio T. The influence of mother-child incompatibility on Rh immunization.Vox Sang 1956;1:26-30.

19. Finn R. Erythroblastosis. Lancet 1960;1:526-531.
20. Clarke CA, Finn R, Mc Connell RB, Sheppard PM. The protection afforded by $A B O$ incompatibility against erythroblastosis due to rhesus antiD. Int Arch Allerg 1968;13:380-392.

21.W ooddrow JC,Finn R.Transplacental haemorrhage. Br J H aematol 1966; 12:297-304

22. Finn R, Clarke CA, D onohoe W, McC onnell RB, Sheppard PM, Lehane $D$, Kulke W. Experimental studies on the prevention of Rh hemolytic disease. Br Med J 1961;1:148-156

23. A combinated study from centers in England and Baltimore. Prevention of haemolytic disease: Results of clinical trial. Br Med J 1966;2:907912.

24. Clarke CA. Prevention of Rh haemolitic disease. Br Med J 1967;4:7-12. 25. FredaVJ, Gorman JG, Pollack W. Rh factor: Prevention of isoimmunization and clinical trial on mothers. Science 1966;151:828-834.

26. Preisler 0 , Schneider J. Idie prophylaxie der sensibilisierung im Rhesus-system. Bibl Gynaec 1966;38:1-22.

27. Zavala C, Payling-W right C. Prevención de la enfermedad hemolítica del recién nacido debida a incompatibilidad del factor Rh(D). Estado actual. Rev Invest C lin 1968;20:209-219.

28. Zavala C, Salamanca F. Mothers at risk of alloimmunization to the Rh (D) antigen and availability of $\mathrm{g}-\mathrm{G}$ lobulin at the Mexican Institute of Social Security.Arch Med Res 1996;27:373-376.

29. Aschmann H.The central desert of Baja California: Demography and Ecology. Berkeley: University of C alifornia Press, 1959

30. A guilar-Marco JL. Misiones en la Península de Baja California. México, D.F.: Instituto N acional de Antropología e Historia, 1991.

31. Clavijero F. H istoria de laAntigua o Baja California. En: Palou F, ed.Vida de Fray Junípero Serra y Misiones de la C alifornia Septemtrional. Estudios preliminares por Miguel León Portilla. México, D.F.: Editorial Porrúa, 1990. 32. Martínez P. Historia de Baja California. La Paz, Baja California, México: Consejo Editorial del Gobierno de Baja California Sur, 1991.

33. Cariño-O lvera $M$. Historia de las relaciones hombre naturaleza en Baja California Sur 1500-1940. Universidad Autónoma de Baja California Sur. México, D.F.: Promarco, 1996.

34. Instituto $\mathrm{N}$ acional de Estadística, Geografía e Informática.A nuario estadístico del estado de Baja California Sur. México, D.F.: IN EGI, 2000:4546.

35. Secretaría de Salud. N orma 0 ficial Mexicana N O M-003-SSA2-1993. Para la disposición de Sangre Humana y sus Componentes con Fines Terapéuticos. Diario 0 ficial de la Federación, 8 de diciembre de 1993.

36. Hardy G. Mendelian proportions in a mixed population. Science 1908: 28-33.

37. Weinberg $W$. Über den nachweis der vererburg beim menschen. Jhrb Ver Vateal N aturk 1908;64:368-382.

38. Ramírez B. N egros, mulatos, indios y piratas en el Golfo de California (época colonial). Memoria del Sexto Simposio de Historia y Antropología Regionales. México, D.F.: Universidad Autónoma de Baja California Sur, 1995:13-17.

39. Reveles-G urrola F. Estudios de polimorfismo genético en el estado de Zacatecas. (Tesis). Monterrey, México; U niversidad Autónoma de N uevo León, 1987.

40. Crawford MH, Lesión W C, Brown K, Less F, Taylor L. Human biology in Mexico. II.A comparison of blood group, serum and red cell enzyme frequencies, and the genetic distances of the Indian populations of Mexico. Am J Phys Anthropol 1974;41:251-268. 